\title{
Mapping the thermo-induced valence tautomerism in Cobalt dioxolane single crystals
}

\author{
Ludmila Leroy ${ }^{1,5}$, Lucas G. Pimenta ${ }^{1}$, Leonardo F. Calazans ${ }^{1}$, Marcos Antônio Ribeiro², Frederico A. Lima ${ }^{3}$, \\ Simone Alexandre ${ }^{1}$, David A. Shultz ${ }^{4}$, Majed Chergui ${ }^{5}$, Carlos B. Pinheiro ${ }^{1}$
}

\author{
${ }^{1}$ Physics department, Universidade Federal de Minas Gerais, 31270-901 Belo Horizonte, MG - Brazil, \\ ${ }^{2}$ Chemistry department, Universidade Federal do Espirito Santo, Espirito Santo, ES - Brazil, \\ ${ }^{3}$ European XFEL, Holzkoppel 4, 22869 Schenefeld, Germany, \\ ${ }^{4}$ Department of Chemistry, North Carolina State University, Raleigh, NC 27695-8204, USA, \\ ${ }^{5}$ Laboratoire de Spectroscopie Ultrarapide, ISIC-FSB-BSP, Ecole Polytechnique Fédérale de Lausanne, \\ CH-1015 Lausanne, Switzerland
}

\section{ludmilaleroy14@ufmg.br}

The development and investigation of smart materials, which present bistability when exposed to external stimuli is a key challenge to material physics and chemistry. Among the various types of these materials, the valence tautomers are compounds which switch between different electronic and spin states and can be used as sensors, signal processors and memory storage [1] since their solid structure does not present substantial rupture during the valence tautomerism (VT) interconversion. The VT has been studied in molecules with a cobalt metal center, nitrogen based ancillary ligands and semiquinone radicals [2-3], and it was observed that it is modulated by the ancillary ligand. For these cobalt complexes, the VT takes place in a reversible fashion [4], in both liquid state and solid state, as single crystals, being possibly dependent on the solid-state arrangement of the complexes and on solvation [5-6]. The VT in such molecules can be induced by temperature as first and second order transitions with a wide range of characteristic $T_{1 / 2}$ according to the ancillary ligand. In the low temperature regime, the VT is also shown to be induced with photo irradiation in multiple wavelengths. Interestingly, it can also be induced with soft and hard X-rays irradiation with high yield of metastable isomers [7-8].

Among the cobalt complexes that display VT, the cobalt 3,5-di-tert-butyl semiquinone pyridine complex is a particularly interesting tautomer, because not only its valence tautomerism can be thermo and photo-induced, but also turned on or off by the presence of solvent molecules in the crystal lattice [5]. It can be crystallized in two different forms, with and without a solvent molecule in the crystal lattice. The first shows no temperature dependence of its magnetic susceptibility, and in the second, the same dependence indicates that only half of the cobalt centers in the unit cell present VT, which we confirmed in X-ray diffraction (XRD) experiments. This, along with results of density functional theory (DFT) calculations, raised an interesting possibility of studying the behavior of particular sites of the crystal separately, utilizing X-ray energies around the cobalt K-edge to understand how each particular site responds to the temperature and how the total VT interconversion takes place within the crystal lattice. In our work we combine the site selectivity of XRD and the characteristic resonant X-ray absorption by cobalt atoms in different oxidation states, in order to spatially map the thermoinduced valence tautomerism within the crystal, and also within the cobalt complexes.

[1] O. Sato, Nat. Chem., vol. 8, no. 7, pp. 644-656, 2016.

[2] D. A. Shultz, Magn. Mol. Mater. II Models Exp., pp. 281-306, 2001.

[3] J. Tao et al., J. Am. Chem. Soc., vol. 128, no. 6, pp. 1790-1791, 2006.

[4] T. Tezgerevska et al., Coord. Chem. Rev., vol. 268, pp. 23-40, 2014.

[5] Y. Mulyana et al., Dalton Trans., vol. 39, no. 20, pp. 4757-4767, 2010.

[6] M. A. Ribeiro et al., Cryst. Growth Des., vol. 16, no. 4, pp. 2385-2393, 2016.

[7] G. Poneti et al, Angew. Chem. Int. Ed., vol. 49, no. 11, pp. 1954-1957, 2010.

[8] T. M. Francisco et al., J. Phys. Chem. Lett., vol. 8, no. 19, pp. 4774-4778, 2017.

Keywords: Valence tautomerism, spatial mapping, cobalt complexes, X-ray diffraction, X-ray absorption 